\title{
The Progress of Next Generation Sequencing in Preimplantation Genetic Testing
}

\section{Beatrice Chung Fat King Brunet ${ }^{1}$, Mohammad Bilaal Toorabally ${ }^{2}$, Wei Wu', Jiayin Liu ${ }^{1 *}$}

${ }^{1}$ The State Key Laboratory of Reproductive Medicine, Clinical Center of Reproductive Medicine, First Affiliated Hospital, Nanjing Medical University, Nanjing 210029, PR China

${ }^{2}$ Department of Cardiology, The First Affiliated Hospital, Nanjing Medical University, Nanjing 210029, PR China;

*Corresponding Author: Jiayin Liu, The State Key Laboratory of Reproductive Medicine, Clinical Center of Reproductive Medicine, First Affiliated Hospital, Nanjing Medical University No. 16 Yongqingxiang Street, Nanjing 210029, PR China, Tel + 86-25-6830-2608(0); E-mail: jyliu_nj@126.com

Received: 08 May 2018; Accepted: 14 May 2018; Published: 16 May 2018

\begin{abstract}
Preimplantation genetic testing (PGT), the determination of an embryo's constituency before implantation, is becoming more and more common in clinic and there has been a gradual shift in technology towards next generation sequencing (NGS). Numerous biopsy samples such as a blastomere of cleavage stage embryo, trophectoderm of blastocyst and polar bodies as well as non-invasive or low-invasive samples such as blastocoele fluid and embryo's culture medium can be analyzed with different NGS platforms namely Roche, Illumina and Ion torrent. NGS technology has been validated and is now clinically applied to detect aneuploidies, partial or segmental aneuploidies, chromosomal aberrations including imbalanced translocations, inversion, deletion, duplication insertion and mosaicism, triploidy and single gene disorder which are often responsible for infertility and can be associated with spontaneous abortions and fetal malformations or diseases. Comprehensive chromosomal screening for all 24 chromosomes together with single gene disorder detection can be achieved at the same time in a single run using NGS and the analysis of multiple samples with different indication can be done on a single chip. NGS has the ability to detect numerical, structural and genetic abnormalities at a low cost with high efficiency and accuracy.
\end{abstract}

Keywords: Next generation sequencing; Preimplantation genetic testing; Preimplantation genetic diagnosis; Preimplantation genetic screening 


\section{Introduction}

Preimplantation genetic testing (PGT) is the determination of an embryo's chromosomal constituency before implantation. It has been given many different names according to its use. Preimplantation genetic diagnosis (PGD) is often used with parents diagnosed with known single gene mutation and chromosome number or structure abnormalities who want to have an unaffected child. Preimplantation genetic screening (PGS) is the screening for aneuploidy especially in patients or couples who have a history of recurrent spontaneous abortions, recurrent implantation failure and advanced maternal age [1]. The goal of all of the above is to have an unaffected child, avoid the termination of pregnancy or recurrent miscarriages.

28 years ago, the first case of a live birth of a normal offspring from PGD for a recessive X chromosome-linked disease was born [2]. Nowadays, PGT is routinely used in clinics with a range of uses including single gene disorder and both structural and numerical chromosomal abnormalities. The success of PGT has led to over 10,000 babies born worldwide.

Since its adoption in clinics, a number of techniques have emerged from the cytogenetic molecular analysis. Fluorescent in situ hybridization (FISH) was the first diagnostic method used. However, FISH has several technical limitations including the number of probes required to obtain reliable results and the requirement of specific parent karyotyping prior to testing [3-5]. It analyzes a limited number of chromosomes at a time and thus misses aneuploidies in other chromosomes. These constraints have led to the development of new and improved 24 chromosomal copy number analysis technologies, including comparative genomic hybridization (CGH), array comparative genomic hybridization (aCGH), digital-PCR, real-time quantitative PCR, SNP microarray and next generation sequencing (NGS), which is the newest method to date. All of these different technologies have their own advantages and disadvantages according to the time of analysis, the cost, the resolution, the labor needed, the procedures and the abnormalities which can be detected ${ }^{1}$. This review is a summary of the advances in preimplantation diagnosis with NGS.

\subsection{Different platforms used in NGS}

There are currently three major NGS platforms, namely Roche (also known as 454), Illumina and Ion torrent. Life technologies/Ion torrent and Roche/454 life sciences, both use emulsion PCR as their amplification method. Illumina on the other hand, uses bridge PCR. As for their sequencing techniques, Roche/454 life sciences use pyrosequencing with a read length of up to $1000 \mathrm{bp}$. But this method has a high error rate in detecting insertions and deletions in homopolymers. Illumina uses sequencing by synthesis with reversible terminator. The newer platforms of Illumina can yield about 24million short fragments of DNA sequence per run or "read" which gives it an ultrahigh-throughput and makes it cost effective. The main limitation of Illumina is in the increased base substitution error rate. Lastly, we have Life technologies/Ion torrent which uses ion semiconductor sequencing and can read up to 200bp. It has high error rates in calling insertions and deletions. Although it takes days to have one genome sequence with Illumina, it is the most widely used on the market due to its cost effectiveness, good sequencing throughput and accuracy [6]. Other emerging sequencing techniques still need work done in terms of cost, quantity, 
quality and time.

\section{Different biopsy sites}

\subsection{Blastomere of cleavage stage embryo VS Trophectoderm of blastocyst}

After fertilization, each cleavage of the zygote yields two undifferentiated cells called blastomeres. For the purpose of PGT, in some centers, Day 3 cleavage cell blastomeres are usually biopsied to be analyzed. Another, most commonly used, stage at which the biopsy is taken, is the blastocyst stage at day 5 or day 6 . Most specifically, cells from the outer layer of the blastocyst are taken, which are known as the trophectoderm after gastrulation. Many researchers dispute over which stage of the embryo yields better outcomes. In 2015, Lukaszuk et al. [7] published a prospective case control study in which the Day 3 blastomeres were used for aneuploidy analysis by NGS in implantation failure patients. Then, fresh day 5 embryo transfer was performed and the clinical pregnancy rates were compared to a control group in which no ploidity status analysis was done. They concluded that the clinical pregnancy rate was more than two times higher in the NGS-blastomere group compared to the control group and the results were statistically significant. But more and more studies are pointing towards and suggesting trophectoderm biopsy as a better choice. Blastomere biopsy has been shown to have several disadvantages. Scott et al. [8,9] have shown that there is a decreased rate of implantation when the biopsy is performed at the blastomere stage. Mertzanidou et al. [10] revealed that at the cleavage stage, mosacism is more likely to be present in the embryos. In 2013, Capalbo et al. [11] showed that mosaicism can be avoided or reduced if the biopsy is done on blastocysts instead of blastomeres. In another study by Scott et al. [9], biopsy performed on the blastomere at the cleavage stage had more damaging effects on the embryos. The results from studies by Jones et al. [12] and Capalbo et al. [13] both show that less genetic material is obtained from biopsy of the embryo at the cleavage stage. The reasons are due to the higher efficiency with trophectoderm biopsy. The likelihood for an euploid embryos to reach the blastocyst stage is higher than that of an abnormal embryo. This decreases the number of biopsies that have to be performed in order to obtain a normal euploid embryo from the mix that will be ultimately used for intrauterine transfer. The positive predictive value is therefore increased when trophectoderm biopsy is used. Lee et al. [14] in a randomized controlled trial and Dahdouh et al. [15] in a metanalysis both demonstrated the better effectiveness of trophectoderm biopsy.

\subsection{Polar bodies}

Treff et al. [16], in 2016, extracted occytes from mouse models and subjected them to in-vitro maturation after which, they separated the polar bodies from the mature occytes. Wide Genome Amplification followed by NGS were performed on the oocytes, polar bodies and blastocysts to check for aneuploidy. They showed that NGS can be applied to gametes and embryos for the chromosome evaluation as long as the genome is known. Furthermore, Yan et al. [17] performed an analysis on both trophectoderm and polar bodies biopsies using NGS and linkage analysis for a single gene disorder PGD, the female patient was a carrier of an X-linked chromosome recessive disorder; $\mathrm{X}$ linked hypohidrotic ectodermal dysplasia and successful live birth was achieved. Thus, showing that NGS can be used for analyzing human polar bodies. 


\subsection{Blastocoele fluid and culture medium}

Recently, non-invasive or low-invasive approaches for chromosomal analysis in PGT have been tried. Several studies mentioned the existence of DNA material including chromosome, genomic and mitochondrial DNA in the blastocoele fluid and culture medium. Xu et al. [18] conducted a study using NGS to analyze fluid from the culture medium of human embryo from day 3 to day 5 and validated their results by comparing it with the comprehensive chromosome sequencing analysis obtained by the corresponding Day 5 whole embryo. Their results showed that non invasive chromosome screening had a specificity of $84 \%$ and a sensitivity of $88.2 \%$ with a positive and negative predictive value of $78.9 \%$ and $91.3 \%$ respectively. Furthermore, using non invasive chromosome screening only, they also carried out the first live birth in a patient with a balanced translocation $\mathrm{t}(14 ; 15)$ (q22; q24) and repeated the procedure on six more patients which resulted in five healthy chromosomally normal offspring and one ongoing pregnancy. Zhang et al. [19] on the other hand, did single gene PGD using NGS on the DNA found in blastocoele fluid, which is routinely removed from the embryo prior to vitrification to prevent damage by ice crystal formation, and compared the results with the corresponding blastomere embryo. They tested ten genes namely TCIRG1, SCN5A, RHO, EXTL1, SLC4A1, VWF, HSF4, NPC1, PTCH1, and EPS8L3.They obtained a gene level amplification efficiency of $84 \%$ which is lower compared to WGA for a single cell which is $90 \sim 95 \%$. There is still room for improvements in the chromosomal analysis and gene level analysis for both fluid culture medium analysis and blastocoele fluid analysis. The source of the DNA should be explained and the results from the blastocoele fluid analysis should be validated. Their non-invasiveness gives these two methods a great potential to make PGT even safer and more routinely used in clinics.

\section{Frequent abnormalities which can be detected}

3.1 Structural abnormalities: Translocation (reciprocal, Robertsonian), inversion, deletion, duplication insertion, ring, marker

Chromosomal aberrations are often responsible for infertility and can be associated with spontaneous abortions and fetal malformations. One of the most common ones is translocation which is a breakage and transfer of the broken part to another location on the same chromosome or most frequently on another chromosome. Two types of translocations can be identified in the process of PGD. They are Robertsonian translocation and reciprocal translocation. Robertsonian translocation involves the breakage at or near the centromere while reciprocal translocation involves the exchange of materials between non homologous chromosomes. Inversions, whereby part of the chromosome is reversed end to end, and insertions, where part of one chromosome is deleted from its normal location and inserted into another chromosome, are also often detected during PGD. Up till now, aCGH remains the gold standard to detect those chromosome aberrations in clinics. Recently, newer methods have emerged to challenge aCGH more specifically, NGS.

In 2013, Yin et al. [20] showed that NGS can be used to detect chromosomal abnormalities. They analyzed the trophectoderm of patients with translocations and inversions using both NGS and SNP array. They also validated their results using qPCR and found that the sensitivity and specificity to detect copy number variance (CNV) was $95 \%$ and $96 \%$ respectively. They also analyzed for aneuploidies and found that NGS sensitivity and specificity were 
both $100 \%$. In 2015, Bono et al. [21] conducted a blinded retrospective study to validate the use of a semiconductor NGS for the detection of reciprocal translocations. They used cells from the blastocyst stage and embryo cleavage cells from patients previously identified with reciprocal translocations for their analyses and compared the comprehensive chromosome analysis results with that obtained from aCGH. They successfully showed that semiconductor NGS is a robust method of detection for unbalanced reciprocal translocations with a specificity of $99.75 \%$ and sensitivity of $100 \%$ at the chromosome identification level. As for the identification of chromosomally normal embryo, both their specificity and sensitivity were $100 \%$. The smallest detectable chromosomal segment was $5 \mathrm{MB}$. Other investigators have also further validated NGS for not only reciprocal translocation but also robertsonian translocations, insertions and inversions. Deleye et al. [22] in 2015, published a paper in the journal of fertility and sterility that compared Illumina and ion torrent (life technologies) NGS to aCGH. They used trophectoderm biopsies at day $5 / 6$ to analyze for numerical and structural chromosome aberrations. The results from NGS were concordant with that of aCGH. The specificity and sensitivity of NGS were both $100 \%$. They reported a smallest detectable chromosomal abnormality of 4.5MB and also that NGS provided an increased signal to noise ratio for lengths up to $3 \mathrm{MB}$. But at lengths lesser than $3 \mathrm{MB}$, the WGA representation bias affects the results. Following these promising results, in 2016, Zhang et al. [23] was the first to apply CNV-seq NGS in clinics to identify chromosomally normal embryos for intrauterine transfer without the assistance of aCGH to validate the embryos biopsies results for patients previously established as having Robertsonian and reciprocal translocations. Due to the shift of trend during the study, they started with cleavage stage biopsy and ultimately changed to blastocyst biopsy. After having been identified as normal/balanced euploid, one or two frozen embryos were transferred. They observed an implantation rate of $80 \%$, a 20 -week gestation clinical pregnancy rate of $60 \%$ and a live birth rate of $70 \%$. They demonstrated that CNV-seq NGS has a high resolution which can detect unbalances near the terminal parts of the $\mathrm{p}$ and $\mathrm{q}$ arms, and can detect mosaicism down to $20 \%$. Also, they suggested that NGS is a reliable and accurate method that has great clinical applications.

For the purpose of PGD, clinicians usually stop at differentiating normal/balanced from chromosomally abnormal/unbalanced aneuploid embryos. But many of those embryos may be carriers of the chromosomal defect and babies from these embryos may in turn have infertility issues and pass on the defect to future generations. Efforts were made to go a step further so as to identify completely chromosomally normal embryos from carrier embryos. In 2016, Hu et al. [24] published a paper in which they used 8 patients with reciprocal translocations. Peripheral blood samples from these patients were used for chromosomal microdissection and PCR amplification followed by NGS to obtain the precise location of the breakpoints. Using this information, they modulated breakpoint specific primers. Trophectoderm biopsies were taken from the embryos of these patients and PGD was done using NGS. The embryos identified as balanced/euploid embryo were further analyzed using linkage analysis with informative SNP and junction spanning PCR analysis to separate the normal embryo from the carrier embryos. For patients who did not have completely normal embryos, the carrier embryos were still transferred. Single or double frozen embryo transfer was done in 8 patients resulting in 6 live births ( 5 balanced translocation carriers and one normal karyotype) with karyotypes corresponding to the PGD. The specificity and sensitivity, and its use in other types of chromosomal aberrations is yet to be tested. 
Furthermore, small deletion and duplication can cause chromosome disease syndromes associated with copy number variance which can lead to severe diseases. With the use of NGS, we can now accurately detect duplication and deletions as small as $1-2 \mathrm{Mb}$. Fan $\mathrm{J}$ et al. [25] validated their resulted using 3 genomic DNA samples with these specific copy number variances and they also used 5 peripheral blood samples from patients with these diseases. They were able to accurately detect a $4.98 \mathrm{Mb} 5 \mathrm{q} 35$.2-qter deletion associated with Sotos syndrome and a $6.66 \mathrm{Mb}$ 7 p22.1-pter deletion associated with 7 p terminal deletion syndrome. Moreover, Gui B et al. [26] was able to successfully detect euploid Charcot-Marie-Tooth disease type A for embryo transfer after NGS-based method using linkage analysis. These results shows that NGS can detect very small copy number variance and can help couples conceive a disease free embryo.

Although there have not yet been any publications on PGD using NGS for ring and marker structural chromosome abnormality, according to the studies mentioned above, NGS has the efficiency and potential to detect them. Thus, further studies are needed to validate the use of NGS for ring and marker chromosome.

\subsection{Aneuploidy}

When the number of chromosome in a cell deviate from the normal chromosomal composition, it is called aneuploidy. It can be due to the addition of a single chromosome (trisomy) or the addition of a pair of chromosomes (tetrasomy) or the loss of a single chromosome (monosomy) or the loss of a pair of chromosomes (nullisomy). Incorrect number of chromosomes in an embryo often lead to implantation failure, miscarriage and fetal malformations. With the development of preimplantation genetic, 24 chromosome screening for aneuploidy is very common now. Fiorentino et al. [27,28] designed a 2 phase study for the development, validation and application of NGS for 24 chromosome aneuploidy screening. During the first phase, they evaluated samples with known abnormal cytogenetic karyotype from cultured amniotic fluid and products of conception, and they re-evaluated previously tested aCGH blastomeres with their NGS-based protocol and any conflicting results were reevaluated using QF-PCR. They showed that NGS had a $99.98 \%$ specificity and a $100 \%$ sensitivity to detect aneuploidy according to chromosome copy number assignment. NGS specificity and sensitivity to detect aneuploidy for 24chromosome diagnosis were both $100 \%$. The positive and negative predictive value of NGS for 24 chromosome aneuploidy screening were both $100 \%$. In the second phase of their study, they analyzed blastocysts prior to embryo transfer using both NGS and aCGH, and conflicting results were reevaluated using QF-PCR. Their results were similar to the first phase and they demonstrated the clinical application of NGS with a clinical pregnancy rate per embryo transfer of $63.8 \%$ and an on-going implantation rate of $62 \%$. For Kung et al. [29] previous blastocyst and cleavage stage embryos which were tested with aCGH were reanalyzed using NGS. They found that NGS specificity and sensitivity were both $100 \%$ and that there was more than $99 \%$ concordance between NGS and aCGH. These results were also confirmed by Zheng et al. [30] using VeriSeq PGS kit (Illumina) with a smallest detection size of 1.8MB and Sachdeva et al. [31] using Ion torrent Personal Genome Machine NGS. After the validation of NGS, its clinical applications in PGS cycles were also evaluated. There is a new targeted next generation sequencing (tNGS) platform which has recently undergone preclinical validation for comprehensive chromosome screening by Zimmerman et al. [32]. They tested the new tNGS platform on both commercially available cell line and discarded 
blastocyst and found the consistency with conventional karyotyping for the cell lines to be $99.2 \%$ and $98.7 \%$ for real-time PCR for the blastocyst. Yang et al. [33] first re- validated NGS by showing specificity, sensitivity, positive and negative predictive value of NGS to be $100 \%$ by reanalyzing embryos which were previously tested with aCGH. Next, they performed a randomized controlled trial with one group using only NGS and the other group using only aCGH. Their results revealed no significant difference in clinical pregnancy, ongoing pregnancy and miscarriage rates between the two groups. Now, NGS is applied in the evaluation of different PGS indication like AMA, RPL and RIF [34].

\subsection{Mosaicism}

Mocaisim in an embryo is defined as having two or more different cells lines with different chromosomal number or structure in one embryo caused by errors in chromosomal segregation during mitosis. At the beginning, when FISH was used for PGS, about 30\% of embryos were found to be mosaic. With recent technologies, new methods for comprehensive chromosome screening (CCS) like aCGH, qPCR and SNP array can detect both euploid and aneuploidy embryos but are unable to detect mosaicism. However high-resolution NGS has the ability to detect euploid and aneuploidy embryos as well as mosaicism. Maxwell et al. [35] internally validated NGS protocols to detect mosaicism. They compared the results from previous euploid embryos which were tested using aCGH and miscarried, to the results of the same embryos using NGS. They found that 31.6\% were mosaic. Both Maxwell et al. [35] and Munne et al. [36] showed that $9 \%$ of all transferred blastocysts leading to ongoing pregnancies were mosaic.

Munne et al. [37] propose that there should be a third and intermediary group for mosaic embryo. According to the Controversies in Preconception, Preimplantation and Prenatal Genetic Diagnosis (COGEN) and Preimplantation Genetic Diagnosis International Society (PGDIS) blastocyst biopsies are now categorized into 3 groups for implantation: euploid embryo ( $<20 \%$ aneuploidy cells), aneuploid embryo ( $>80 \%$ aneuploidy cells) and euploidaneuploid mosaics (20\%-80\% aneuploidy). Although the latter often miscarries and implants less frequently, about $40 \%$ of mosaic embryo can lead to live birth [38,39]. Munne et al. [36] retested and analyzed the results using highresolution NGS from previous PGS cycle that used aCGH and divided them into two groups: mosaic group and euploid group. They found that even though the euploid group (71\%) had a higher implantation rate than the mosaic group (53\%), the difference was insignificant. They also showed that the mosaic embryos do miscarry and implant less, however they discovered that complex mosaic embryos have a significantly lower implantation rate (10\%) compared to other mosaic embryos. They proposed that embryos with $20 \%-40 \%$ mosaicism should be selected first instead of embryos with $>40 \%$ mosaicism in accordance with COGEN guidelines. Since complex mosaic embryos had a $10 \%$ implantation rate, they proposed that the latter could be used in patients with advanced maternal age and poor reproductive history. PGDIS proposed guideline suggest that monosomic embryos should be prioritized over trisomic embryos, though Munne et al. [36] presented that trisomic, monosomic or segmental mosaics had similar implantation rates. Munne et al. [36] also suggested more research is needed to validate the other recommendations made by COGEN and PGDIS regarding which mosaic embryo should be transferred according to the particular chromosomes involved. Caution should be taken when proposing the transfer of mosaic embryo, if for the first cycle 
only one mosaic embryo is found, then the patient should perform a second cycle and if the second cycle is also unsuccessful, then the patient should consider the transfer of a mosaic embryo. The patients should be offered different options along with the associated risk and advantages so that an informed decision can be made.

\subsection{Triploidy}

Triploidy is a rare chromosomal disorder which affects $2-3 \%$ of human pregnancies. It is defined as a polypoid with three sets of chromosomes. Triploidy in humans are 69XXX, 69XXY, and 69XYY. According to its etiology, it is usually classified into two groups: 1 . Digyny which is when the additional haploid is maternal, 2. Diandry which is when the additional haploid is paternal. It is associated with multiple abnormalities, partial hydatidiform mole and miscarriages. Maxwell et al. [35] internally validated NGS protocols which was able to detect different triploidies (69 XYY and $69 \mathrm{XXY}$ ) which aCGH could not. But Xu et al. [40] found that MALBAC-NGS was not able to detect triploidy compared to SNP array. Recently, Marin et al. [41] validated triploidy detection in blastocyst using tNGS. They were able to accurately detect triploidy in their control samples as well as in trophectoderm biopsies and found that triploidy during in vitro fertilization is a rare occurrence and is mainly linked to maternal origin.

\subsection{Single gene disorder}

Single gene disorder, also known as monogenic disease, is when a single mutation in a specific gene leads to a hereditary disease which can occur early during childhood or have a late onset. Usually these disorders are debilitating, lowers patients' quality of life and sometimes may even be associated with a shorter life span. With the use of PGD, we can now select embryo which are free from these mutations. In fact, in 1992, the first baby free from cystic fibrosis was born after PGD and since then PGD has been increasingly used to detect monogenic disorders [42]. NGS-based PGD for monogenic diseases was first validated by Treff et al. [43] They showed that the genotype obtained with NGS was $100 \%$ consistent with Taqman allelic discrimation test as well as their reference laboratory genotypes for CF: cystic fibrosis and WWS: Wlaker-Warburg syndrome. Then Well et al. [44] showed that low-pass whole genome amplification (WGA) and linkage polymorphism before NGS can detect cystic fibrosis mutation. Together with Fragouli et al. [45], they both showed that the whole mitochondrial genome can be analyzed using NGS. Furthermore, live births were achieved after the use of NGS. Yan et al. [17] described two couples, one with a family history of autosomal dominant disorder, hereditary multiple exostoses (HME), who had trophectoderm biopsies and the other female patient was an X-linked chromosome recessive disorder carrier; Xlinked hypohidrotic ectodermal dysplasia and had a trophectoderm and polar bodies biopsies. Both couples underwent PGD using NGS and linkage analysis. The results were confirmed by Sanger sequencing, CGH array and STR analyses. The two couples successfully gave birth to healthy babies. Chen et al. [46] also published an NGSbased SNP haplotyping method for $\mathrm{Hb} \mathrm{H}$ disease. They ran the same samples in both Gap-PCR and NGS-based SNP haplotyping, the latter was able to obtain results for all the samples while Gap-PCR was only able to diagnose one sample. Chen et al. [46] also achieved a live birth.

All the NGS based method used for monogenic diseases use linkage analysis to obtain their results and require prior analysis of the parents lymphocytes and other reference laboratory samples to acquire a locus-specific product. 
Although this process may require more time and can increase the cost of PGD, single gene disorder screening and comprehensive chromosome screening for aneuploidies can be performed on a single chip and for several samples at one time, thereby reducing its cost.

\section{Embryo transfer}

After NGS analysis, which can take a minimum of 24 hours, either fresh embryo or frozen embryo transfer can be performed. Most clinics perform frozen embryo transfer because NGS is more cost effective when multiple samples are loaded on a single chip and waiting for the appropriate number of samples requires the vitrification of the samples. A randomized controlled trial performed by Coated et al. [47] compared fresh embryo transfer cycles to frozen embryo transfer cycles. They found a significant increase in ongoing pregnancies and live birth rate per intended treatment as well as per embryo transfer in the frozen group. Their results also showed that for double embryo transfer, the live birth rate was significantly increased in the frozen embryo transfer group. Frozen embryo transfer has the advantage of both being cost effective and patients are able to achieve their targeted embryo transfer strategy. Thus preventing OHSS and transferring the embryo into a uterus with the best environment for implantation.

\section{Limitation and efficiency}

NGS has the ability to detect aneuploidies, partial or segmental aneuploidies, chromosomal aberrations including imbalanced translocations, inversion, deletion, duplication insertion and mosaicism, triploidy, single gene disorder and even mitochondrial disorders and its detection capability is more precise and accurate than aCGH with the smallest detection size of $1.8 \mathrm{MB}[48,30]$. It is the only technology which can accurately detect mosaicism. Comprehensive chromosomal screening for all 24 chromosomes together with single gene disorder detection can be achieved at the same time in a single run using NGS and the analysis of multiple samples with different indication can be done on a single chip which reduces the cost [49]. NGS is highly automated and has a high-throughtput which further reduces the cost. The time for analysis has been shortened and can now be used in fresh cycles, however for cost saving to allow it to run to its full capacity, frozen cycles are more commonly performed.

One of the limitations of NGS is that it cannot detect triplet repeat disorder such as fragile X syndrome. For single gene disorder detection, the phenomenon of allele drop out may occur and may lead to erroneous results. Therefore, linkage analysis needs to be performed together with NGS to achieve positive results [50].

\section{Conclusion}

With preimplantation genetic testing (PGT) becoming more and more common in clinic nowadays and with the gradual shift in technology towards next generation sequencing (NGS) which has the ability to detect numerical, structural and genetic abnormalities at a low cost, highly automated, high-throughtput and improves efficiency and accuracy. 


\section{Funding details}

None

\section{Acknowledgements}

None

\section{Conflict of interests}

The authors declare that there is no conflict of interest

\section{References}

1. Handyside AH. 24-chromosome copy number analysis: a comparison of available technologies. Fertil Steril 100 (2013): 595-602.

2. Handyside AH, Kontogianni EH, Hardy K, Winston RM. Pregnancies from biopsied human preimplantation embryos sexed by Y-specific DNA amplification. Nature 344 (1990): 768-770.

3. Munné S. Preimplantation genetic diagnosis of numerical and structural chromosome abnormalities. Reprod Biomed Online 4 (2002): 183-196.

4. Velilla E, Escudero T, Munné S. Blastomere fixation techniques and risk of misdiagnosis for preimplantation genetic diagnosis of aneuploidy. Reprod Biomed Online 4 (2002): 210-217.

5. DeUgarte CM, Li M, Surrey M, Danzer H, Hill D, DeCherney AH. Accuracy of FISH analysis in predicting chromosomal status in patients undergoing preimplantation genetic diagnosis. Fertil Steril 90 (2008): 1049-1054.

6. Xuan J, Yu Y, Qing T, Guo L, Shi L. Next-generation sequencing in the clinic: promises and challenges. Cancer Lett 340 (2013): 284-295.

7. Łukaszuk K, Pukszta S, Wells D, Cybulska C, Liss J, Płóciennik Ł, et al. Routine use of next-generation sequencing for preimplantation genetic diagnosis of blastomeres obtained from embryos on day 3 in fresh in vitro fertilization cycles. Fertil Steril 103 (2015): 1031-1036.

8. Scott RT, Upham KM, Forman EJ, Hong KH, Scott KL, et al. Blastocyst biopsy with comprehensive chromosome screening and fresh embryo transfer significantly increases in vitro fertilization implantation and delivery rates: a randomized controlled trial. Fertil Steril 100 (2013): 697-703.

9. Scott RT, Upham KM, Forman EJ, Zhao T, Treff NR. Cleavage-stage biopsy significantly impairs human embryonic implantation potential while blastocyst biopsy does not: a randomized and paired clinical trial. Fertil Steril 100 (2013): 624-630.

10. Mertzanidou A, Wilton L, Cheng J, Spits C, Vanneste E, Moreau Y, Vermeesch JR, Sermon K. Microarray analysis reveals abnormal chromosomal complements in over $70 \%$ of 14 normally developing human embryos. Hum Reprod 28 (2013): 256-264.

11. Capalbo A,Wright G, Elliott T, Ubaldi FM, Rienzi L, Nagy ZP. FISH reanalysis of inner cell mass and trophectoderm samples of previously array-CGH screened blastocysts shows high accuracy of diagnosis 
and no major diagnostic impact of mosaicism at the blastocyst stage. Hum Reprod 28 (2013): 2298-2307.

12. Jones GM, Cram DS, Song B, Kokkali G, Pantos K, Trounson AO. Novel strategy with potential to identify developmentally competent IVF blastocysts. Hum Reprod 23 (2008): 1748-1759.

13. Capalbo A, Ubaldi FM, Cimadomo D, Maggiulli R, Patassini C, Dusi L, Sanges F, Buffo L, Venturella R, Rienzi L. Consistent and reproducible outcomes of blastocyst biopsy and aneuploidy screening across different biopsy practitioners: a multicentre study involving 2586 embryo biopsies. Hum Reprod 31 (2015): 199-208.

14. Lee E, Illingworth $\mathrm{P}$, Wilton L, Chambers GM. The clinical effectiveness of preimplantation genetic diagnosis for aneuploidy in all 24 chromosomes (PGD-A): systematic review. Hum Reprod 30 (2015): 473-483.

15. Dahdouh EM, Balayla J, Garc1'a-Velasco JA. Impact of blastocyst biopsy and comprehensive chromosome screening technology on preimplantation genetic screening: a systematic review of randomized controlled trials. Reprod Biomed Online 30 (2015): 281-289.

16. Treff NR, Krisher RL, Tao X, Garnsey H, Bohrer C, Silva E, et al. Next Generation Sequencing-Based Comprehensive Chromosome Screening in Mouse Polar Bodies, Oocytes, and Embryos. Biol Reprod 94 (2016): 76.

17. Yan L, Huang L, Xu L, Huang J, Ma F, Zhu X, et al. Live births after simultaneous avoidance of monogenic diseases and chromosome abnormality by next-generation sequencing with linkage analyses. Proc Natl Acad Sci 112 (2015): 15964-15969.

18. Xu J, Fang R, Chen L, Chen D, Xiao JP, Yang W, et al. Noninvasive chromosome screening of human embryos by genome sequencing of embryo culture medium for in vitro fertilization. Proc Natl Acad Sci 113 (2016): 11907-11912.

19. Zhang Y, Li N, Wang L, Sun H, Ma M, Wang H, et al. Molecular analysis of DNA in blastocoele fluid using next-generation sequencing. J Assist Reprod Genet 33 (2016): 637-645.

20. Yin X, Tan K, Vajta G, Jiang H, Tan Y, Zhang C, et al. Massively parallel sequencing for chromosomal abnormality testing in trophectoderm cells of human blastocysts. Biol Reprod 88 (2013): 69.

21. Bono S, Biricik A, Spizzichino L, Nuccitelli A, Minasi MG, Greco E, et al. Validation of a semiconductor next-generation sequencing-based protocol for preimplantation genetic diagnosis of reciprocal translocations. Prenat Diagn 35 (2015): 938-944.

22. Deleye L, Dheedene A, De Coninck D, Sante T, Christodoulou C, Heindryckx B, et al. Shallow whole genome sequencing is well suited for the detection of chromosomal aberrations in human blastocysts. Fertil Steril 104 (2015): 1276-1285.e1.

23. Zhang W, Liu Y, Wang L, Wang H, Ma M, Xu M, et al. Clinical application of next-generation sequencing in preimplantation genetic diagnosis cycles for Robertsonian and reciprocal translocations. J Assist Reprod Genet 33 (2016): 899-906.

24. Hu L, Cheng D, Gong F, Lu C, Tan Y, et 1. Reciprocal Translocation Carrier Diagnosis in Preimplantation Human Embryos. EBioMedicine 14 (2016): 139-147.

25. Fan J, Wang L, Wang H, Ma M, Wang S, Liu Z, et al. The clinical utility of next-generation sequencing for 

identifying chromosome disease syndromes in human embryos. Reprod Biomed Online 31 (2015): 62-70.

26. Gui B, Yang P, Yao Z, Li Y, Liu D, Liu N, et al. A New Next-Generation Sequencing-Based Assay for Concurrent Preimplantation Genetic Diagnosis of Charcot-Marie-Tooth Disease Type 1A and Aneuploidy Screening. J Genet Genomics 43 (2016): 155-159.

27. Fiorentino F, Biricik A, Bono S, Spizzichino L, Cotroneo E, et al. Development and validation of a nextgeneration sequencing-based protocol for 24-chromosome aneuploidy screening of embryos. Fertil Steril 101 (2014): 1375-1382.

28. Fiorentino F, Bono S, Biricik A, Nuccitelli A, Cotroneo E, Cottone G, et al. Application of next-generation sequencing technology for comprehensive aneuploidy screening of blastocysts in clinical preimplantation genetic screening cycles. Hum Reprod 29 (2014): 2802-2813.

29. Kung A, Munné S, Bankowski B, Coates A, Wells D. Validation of next-generation sequencing for comprehensive chromosome screening of embryos. Reprod Biomed Online 31 (2015): 760-769.

30. Zheng H, Jin H, Liu L, Liu J, Wang WH. Application of next-generation sequencing for 24-chromosome aneuploidy screening of human preimplantation embryos. Mol Cytogenet 16 (2015): 38.

31. Sachdeva K, Discutido R, Albuz F, Almekosh R, Peramo B. Validation of Next-Generation Sequencer for 24-Chromosome Aneuploidy Screening in Human Embryos. Genet Test Mol Biomarkers 21 (2017): 674680 .

32. Zimmerman RS, Tao X, Marin D, Werner MD, Hong KH, Lonczak A, Landis J, Taylor D, Zhan Y, Scott RT Jr., Treff NR. Preclinical validation of a targeted next generation sequencing-based comprehensive chromosome screening methodology in human blastocysts. Mol Hum Reprod 24 (2018): 37-45.

33. Yang Z, Lin J, Zhang J, Fong WI, Li P, Zhao R, et al. Randomized comparison of next-generation sequencing and array comparative genomic hybridization for preimplantation genetic screening: a pilot study. BMC Med Genomics 23 (2015): 30.

34. Lukaszuk K, Jakiel G, Kuczynski W, Pukszta S, Liss J, Plociennik L, et al. Next generation sequencing for preimplantation genetic testing of blastocysts aneuploidies in women of different ages. Ann Agric Environ Med 23 (2016): 163-166.

35. Maxwell SM, Colls P, Hodes-Wertz B, McCulloh DH, McCaffrey C, Wells D, et al. Why do euploid embryos miscarry? A case-control study comparing the rate of aneuploidy within presumed euploid embryos that resulted in miscarriage or live birth using next-generation sequencing. Fertil Steril 106 (2016): 1414-1419.e5

36. Munné S, Blazek J, Large M, Martinez-Ortiz PA, Nisson H, Liu E, et al. Detailed investigation into the cytogenetic constitution and pregnancy outcome of replacing mosaic blastocysts detected with the use of high-resolution next-generation sequencing. Fertil Steril 108 (2017): 62-71.e8.

37. Munne S, Grifo J, Wells D. Mosaicism: "survival of the fittest" versus " no embryo left behind". Fertil Steril 105 (2016): 1146-1149.

38. Greco E, Minasi MG, Fiorentino F. Healthy babies after intrauterine transfer of mosaic aneuploid blastocysts. N Engl J Med 373 (2015): 2089-2090.

39. Fragouli E, Alfarawati S, Spath K, Tarozzi N, Borini A, Wells D. Analysis of implantation and ongoing 
pregnancy rates following the transfer of mosaic diploid-aneuploid blastocysts. Hum Genet 136 (2017): 805 .

40. Xu J, Niu W, Peng Z, Bao X, Zhang M, Wang L, et al. Comparative study of single-nucleotide polymorphism array and next generation sequencing based strategies on triploid identification in preimplantation genetic diagnosis and screen. Oncotarget 7 (2016): 81839-81848.

41. Marin D, Zimmerman R, Tao X, Zhan Y, Scott RT Jr, Treff NR. Validation of a targeted next generation sequencing-based comprehensive chromosome screening platform for detection of triploidy in human blastocysts. Reprod Biomed Online 36 (2018): 388-395.

42. Handyside AH, Lesko JG, Tarín JJ, et al. Birth of a normal girl after in vitro fertilization and preimplantation diagnostic testing for cystic fibrosis. N Engl J Med 327 (1992): 905e9.

43. Treff NR, Fedick A, Tao X, Devkota B, Taylor D, et al. Evaluation of targeted next-generation sequencingbased preimplantation genetic diagnosis of monogenic disease. Fertil Steril 99 (2013): 1377-1384.e6.

44. Wells D, Kaur K, Grifo J, Glassner M, Taylor JC, et al. Clinical utilisation of a rapid low-pass whole genome sequencing technique for the diagnosis of aneuploidy in human embryos prior to implantation. $\mathrm{J}$ Med Genet 51 (2014): 553-562.

45. Fragouli E, Spath K, Alfarawati S, Kaper F, Craig A, et al. Altered levels of mitochondrial DNA are associated with female age, aneuploidy, and provide an independent measure of embryonic implantation potential. PLoS Genet 11 (2015): e1005241.

46. Chen L, Diao Z, Xu Z, Zhou J, Yan G, Sun H. The clinical application of NGS-based SNP haplotyping for PGD of Hb H disease. Syst Biol Reprod Med 63 (2017): 212-217.

47. Coates A, Kung A, Mounts E, Hesla J, Bankowski B, et al. Optimal euploid embryo transfer strategy, fresh versus frozen, after preimplantation genetic screening with next generation sequencing: a randomized controlled trial. Fertil Steril 107 (2017): 723-730.e3.

48. Yang Z, Lin J, Zhang J, Fong WI, et al. Randomized comparison of next-generation sequencing and array comparative genomic hybridization for preimplantation genetic screening: a pilot study. BMC Med Genomics 8 (2015): 30.

49. Knapp M, Stiller M, Meyer M. Generating barcoded libraries for multiplex high-throughput sequencing. Methods Mol Biol 840 (2012): 155-170.

50. Preimplantation Genetic Diagnosis International Society (PGDIS). Guidelines for good practice in PGID: program requirements and laboratory quality assurance. Reprod Biomed Online 16 (2008): 134-174.

Citation: Beatrice Chung Fat King Brunet, Mohammad Bilaal Toorabally, Wei Wu, Jiayin Liu. The Progress of Next Generation Sequencing in Preimplantation Genetic Testing. Archives of Clinical and Biomedical Research 2 (2018): 132-144.

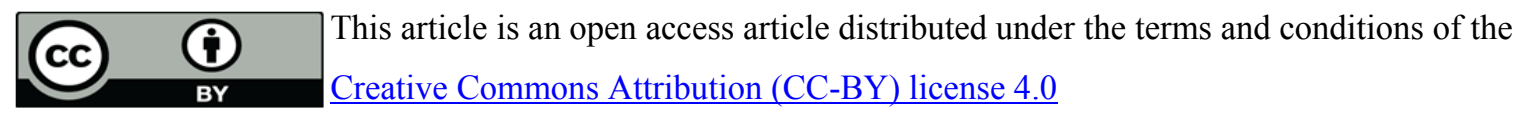

